\title{
Estrategias para mejorar el seguimiento del Programa de Evaluación Auditiva Neonatal Universal
}

\author{
Strategies to improve follow-up of the \\ Universal Newborn Hearing Screening Program
}

Karin Krauss $\mathbf{M}^{1}$, Claudia Heider C $^{1}$, Mónica Sierra G² , Gloria Ribalta L $^{3}$.

\begin{abstract}
RESUMEN
Introducción: El Programa de Evaluación Auditiva Neonatal (PEAN) en Clínica Las Condes se inició el 2001 y ha evaluado más de 18.000 niños. Nuestro desafío actual es lograr que aquellos cuyo examen se encuentre alterado reciban precozmente evaluación y derivación oportuna.

Objetivo: Analizar el impacto de estrategias para mejorar el seguimiento del programa por medio de la reducción del porcentaje de pérdida de seguimiento.

Material y método: Durante 2010 se implementaron nueve estrategias para mejorar el seguimiento del PEAN. Mediante una cohorte retrospectiva se evaluaron los resultados de PEAN 12 meses previos y posteriores a la implementación de éstas.

Resultados: El 2009 se evaluaron 1.797 niños, completaron el protocolo $71,1 \%$, perdiéndose en el seguimiento 28,9\%. El 2011, posterior a la implementación de las medidas, se evaluaron 1.823 niños, completaron el protocolo 85,1\%, perdiéndose en el seguimiento sólo 14,9\%. La diferencia del porcentaje de pérdidas de seguimiento entre el 2009 y 2011 fue de $13.87 \%$ (p: 0.042).

Discusión y conclusiones: Es necesario descubrir las causas de pérdida de seguimiento para establecer estrategias que ayuden a mejorar estas cifras y permitir que todo niño pesquisado sea diagnosticado a tiempo. La implementación de estrategias como las planteadas en este trabajo son una forma práctica y efectiva para mejorar el seguimiento deI PEAN.
\end{abstract}

Palabras clave: Hipoacusia, pérdida de seguimiento, tamizaje neonatal.

\footnotetext{
ABSTRACT

Introduction: The Newborn Hearing Screening Program (NHSP) at Clinica Las Condes began in 2001 and up to date, has evaluated over 18.000 children. Our current challenge is to achieve that children with referred screening, receive early evaluation and intervention.
}

\footnotetext{
${ }^{1}$ Médico Cirujano en Programa de Capacitación en Otorrinolaringología, Clínica Las Condes.

2Tecnólogo Médico, Departamento de Otorrinolaringología, Clínica Las Condes.

${ }^{3}$ Médico Otorrinolaringólogo, Clínica Las Condes.
} 
Aim: To analyze the impact of strategies to improve program's follow-up reducing the percentage of follow-up losses.

Material and method: During 2010 nine NHSP strategies were implemented to improve the follow-up. By a retrospective cohort NHSP's results were evaluated 12 months previous and after the implementation of these strategies.

Results: In 20091.797 children were evaluated, 71,1\% completed the protocol, with 28,9\% lost in the follow-up. In 2011, after strategies' implementation, 1.823 children were evaluated, $85,1 \%$, completed the protocol and only $14,9 \%$ were lost in the follow-up. The difference in the percentage lost between 2009 and 2011 was $13.87 \%$ (p: 0.042).

Discussion and conclusions: It is necessary to discover the reasons of loss to followup, in order to establish strategies that may allow every referred child to be diagnosed in time. Implementation of strategies like the raised in this investigation is a practical and effective way to improve the follow-up of NHSP.

Key words: Hearing Loss, Lost to Follow-Up, Neonatal Screening.

\section{INTRODUCCIÓN}

La implementación de programas de evaluación auditiva neonatal eficientes han logrado mejorar significativamente la pesquisa de recién nacidos con hipoacusia congénita, facilitando así una intervención temprana que permita prevenir las consecuencias cognitivas, verbales, emocionales, educacionales y sociales propias de la enfermedad ${ }^{1}$.

Desde el año 1994, el Joint Committee on Infant Hearing, organismo americano encargado de confeccionar recomendaciones para la identificación temprana de niños con hipoacusia, declaraba la necesidad de realizar una evaluación auditiva neonatal universal ${ }^{2}$. A partir de ese entonces, las nuevas declaraciones se han enfocado en ir mejorando estos programas, de forma que el total de los recién nacidos puedan ser evaluados y más aún, que las intervenciones se realicen precozmente en el intento de evitar las complicaciones antes mencionadas ${ }^{3,4}$.

En Clínica Las Condes (CLC), el programa de evaluación auditiva neonatal se inició en el año 2001 y hasta la fecha ha evaluado a más de 18.000 niños, alcanzando desde el año 2007 una cobertura del $100 \%$ de los recién nacidos vivos en la clínica. Nuestro desafío actual se enfoca en lograr que aquellos recién nacidos cuya evaluación inicial se encuentre alterada reciban precozmente una segunda evaluación, diagnóstico y derivación oportuna.

Cifras del Joint Committee on Infant Hearing indican que en Estados Unidos se realiza tamizaje auditivo a aproximadamente el $95 \%$ de los recién nacidos; de ese $95 \%$, cerca del $2 \%$ tienen una evaluación alterada, pero más de la mitad de estos recién nacidos, no tienen un seguimiento adecuado para confirmar una pérdida auditiva 0 iniciar una intervención temprana ${ }^{4}$.

Estas cifras no son ajenas a nuestra realidad, en una cohorte de recién nacidos de muy bajo peso evaluados en un hospital público de nuestro país se estimó que sólo el 53,6\% de los recién nacidos recibieron seguimiento ${ }^{5}$. En nuestro centro el año 2007 se estimó que la pérdida de seguimiento de aquellos recién nacidos evaluados entre 2001 y 2007, y que tenían un tamizaje alterado alcanzaba a más del $14 \%^{6}$. Por este motivo, el grupo de expertos a cargo del programa se reunió con el objetivo de generar estrategias que permitieran reducir esta cifra, para que la totalidad de los recién nacidos con sospecha de hipoacusia puedan ser diagnosticados y tratados precozmente.

\section{OBJETIVO}

El objetivo del presente trabajo es por tanto analizar el impacto de las estrategias para mejorar el seguimiento del programa por medio de la reducción del porcentaje de pérdida de seguimiento.

\section{MATERIAL Y MÉTODO}

El grupo de expertos de Clínica Las Condes a cargo del PEAN está compuesto por otorrinolarin- 
gólogos, audiólogos y tecnólogos médicos. Luego de evaluar los resultados del programa hasta el año 2009 se constata que existe una importante pérdida en el seguimiento de los pacientes, motivo por el cual se decidió idear estrategias que permitieran mejorar el seguimiento de aquellos niños que ingresaban al programa (Tabla 1). Estas medidas fueron implementadas durante el año 2010 y para evaluar su impacto en la reducción del porcentaje de pérdida de seguimiento, se estudió una cohorte retrospectiva, a través de la base de datos del programa 12 meses previos y 12 meses posteriores a la implementación. La significancia estadística de dicha diferencia porcentual fue evaluada mediante el test de Chi2 de Pearson y se consideró significativo un valor $p<0,05$.

Los pacientes evaluados en nuestro centro se dividen en dos grupos, recién nacidos en sala cuna y recién nacidos de unidad de tratamiento intermedios neonatal. Los padres o tutores responsables de cada recién nacido firman previamente un consentimiento informado para la realización de todas las pruebas de tamizaje neonatal (metabólico, hormonal y auditivo), el cual cuenta con el respaldo del Comité de Ética de Clínica Las Condes. Cada grupo ingresa a un protocolo específico que se describe a continuación:

\section{Protocolo Tamizaje Auditivo Neonatal Universal en CLC}

- Recién nacidos sin factores de riesgo de hipoacusia: Sala cuna:

Fueron evaluados con Emisiones Oto-Acústicas (EOA) por Productos de Distorsión (PD) mediante el equipo GSI AUDIOscreener ${ }^{\circledR}$, después de las 36 horas de vida. Las frecuencias estudiadas fueron 2, 3, 4 y $5 \mathrm{kHz}$, cada una estimulada con tonos de $65 \mathrm{~dB} \mathrm{SPL}$ (L1) y $55 \mathrm{~dB}$ SPL (L2). Los criterios de aprobación del equipo son una relación señal/ruido mayor o igual a $6 \mathrm{~dB}$ y el pasar al menos 3 de las 4 frecuencias.

Cuando el paciente reprueba el tamizaje, es reevaluado nuevamente al mes de vida para repetir las EOA por PD. Lo que fue definido como el primer control posterior al tamizaje.

Si el paciente no pasa este control, es derivado a estudio audiológico completo con Potenciales Evocados Auditivos de Tronco Diagnósticos (PEATD), también conocidos como BERA o ABR clínicos, e impedanciometría. Esta evaluación se consideró como segundo control.

Finalmente si este último estudio resulta alterado, diagnosticando una hipoacusia sensorioneural (onda $V$ por sobre los $25 \mathrm{~dB}$, utilizando tonos click (2 a 4 KHz)), el paciente es enviado para evaluación por otorrinolaringólogo, tercer control.

- Recién nacidos con factores de riesgo de hipoacusia: UTI Neonatología

Fueron evaluados con PEAT automatizados mediante el equipo GSI AUDIOscreener ${ }^{\circledR}$. Para ello se utilizó tono click de rarefacción a $35 \mathrm{~dB}$ HL con criterio Pass de Fsp mayor o igual a 3,2.

Si el paciente reprueba este tamizaje, es derivado directamente a estudio audiológico con PEAT-D, EOA por PD e impedanciometría. Lo que fue considerado como primer control posterior al tamizaje.

Si este último estudio diagnóstico resulta alterado, obteniendo un registro de onda $\mathrm{V}$ por sobre

\section{Tabla 1. Medidas para mejorar el seguimiento del Programa de Evaluación Auditiva Neonatal Universal} en Clínica Las Condes

\begin{tabular}{|c|l|}
\hline 1. & Asignar a una persona que se encargue específicamente del seguimiento de los pacientes en el programa. \\
\hline 2. & Revisar la ficha de los pacientes que reprueben el tamizaje y constatar que exista en ella registro de la evaluación. \\
\hline 3. & Mantener actualizados los datos de ambos padres del paciente (teléfono, dirección, correo electrónico). \\
\hline 4. & Llamar permanentemente a los padres para recordarles que deben acudir a control. \\
\hline 5. & Mantener actualizada una base de datos con los pacientes reprobados. \\
\hline 6. & Si el paciente no asiste a control, se informará a su pediatra, para que esté en conocimiento de la situación. \\
\hline 7. & Programar reuniones mensuales con el Comité de Hipoacusia para evaluar cómo marcha el seguimiento. \\
\hline 8. & Programar reuniones periódicas con neonatología y pediatría para informar de pacientes reprobados. \\
\hline 9. & Implementación de ficha electrónica en Clínica Las Condes. \\
\hline
\end{tabular}


los $30 \mathrm{~dB}$, utilizando tonos click (2 a $4 \mathrm{KHz}$ ) y EOA por PD negativas, el paciente es enviado para evaluación por otorrinolaringólogo, completando un segundo control.

\section{RESULTADOS}

Durante 2009 se evaluaron 1.797 niños, 1.689 correspondientes a sala cuna, 108 a UTI. Del total de niños evaluados, 59 reprobaron el tamizaje lo que corresponde al 3,2\% (30 niños de sala cuna, lo que corresponde al $1,77 \%$ del total de ese grupo y 29 niños de UTI, $26,8 \%$ del total de UTI). De los 59 referidos, completaron el protocolo correspondiente 42 niños $(71,2 \%)$, perdiéndose en el seguimiento 17 niños (28,8\%) (Tabla 2).

El año 2011 se evaluaron 1.823 niños, 1.714 correspondientes a sala cuna, 109 a UTI. Del total de niños evaluados, 87 reprobaron el tamizaje lo que corresponde al 4,7\% (54 niños que corresponde a $3,1 \%$ de sala cuna. 33 niños que corresponde a $30,2 \%$ de UTI). De los 87 referidos, completaron el protocolo correspondiente 74 niños (85\%), perdiéndose en el seguimiento sólo 13 niños (15\%) (Tabla 2).

\section{Tabla 2. Comparación en el seguimiento del PEAN} años 2009-2011

\begin{tabular}{|c|c|c|c|}
\hline & $\begin{array}{c}\text { Reprueba } \\
\text { tamizaje }\end{array}$ & $\begin{array}{c}\text { Completa } \\
\text { protocolo }\end{array}$ & $\begin{array}{c}\text { Pérdida } \\
\text { seguimiento }\end{array}$ \\
\hline 2011 & $4,77 \%$ & $85,05 \%$ & $14,95 \%$ \\
\hline 2009 & $3,28 \%$ & $71,18 \%$ & $28,82 \%$ \\
\hline
\end{tabular}

PEAN: Programa Evaluación Auditiva Neonatal.

La pérdida de pacientes posterior a la aplicación de las medidas descritas disminuyó significativamente de $28,8 \%$ a $15 \%$ (p: 0,42).

\section{DISCUSIÓN}

La problemática del seguimiento es una dificultad común en todo programa o protocolo que evalúe pacientes en forma prospectiva. Es así como en la literatura se describen cifras que van desde $50 \%$ de pérdida en el seguimiento del tamizaje auditivo universal en recién nacidos en un grupo americano multicéntrico ${ }^{7}, 17 \%$ en el grupo del Parkland Memorial Hospital en Texas ${ }^{8}$ a $11 \%$ en el grupo de Massachusetts9, cifras que en nuestro país aún no se pueden estimar dado la inexistencia de un programa nacional de tamizaje universal de hipoacusia.

Identificar la 0 las causas de las pérdidas permite reestructurar dichos protocolos de forma que éstos sean lo más eficiente posible, tarea compleja que requiere de tiempo y dedicación. En nuestra experiencia creemos que uno de los puntos fundamentales para aumentar la eficiencia de los programas es la constante comunicación entre el equipo multidisciplinario. Teniendo en cuenta distintos puntos de vista se puede lograr un trabajo cohesionado que avance en pro de una atención integral. La implementación de estas nuevas estrategias busca en forma escalonada abarcar todas las áreas de probable fuga y para ello la primera medida es fundamental. Consideramos que el hecho de poner un profesional a cargo del seguimiento simplificará de manera consistente las acciones a seguir y seguramente será una estrategia costo efectiva, medida que deberá ser confirmada con las reevaluaciones del efecto que estas intervenciones sigan teniendo en el futuro en nuestra tasa de seguimiento.

Otro punto fundamental es la comunicación fluida con los departamentos de pediatría y neonatología, esto debido a que durante el camino al diagnóstico, serán estos profesionales los "médicos de cabecera" del paciente, quienes deberán reforzar la educación e insistir en la importancia de la evaluación precoz.

Desde el año 2007 el Joint Committee on Infant Hearing planteaba la necesidad de mejorar el seguimiento de los programas, considerando que aproximadamente la mitad de los niños referidos se perdía en el seguimiento ${ }^{7}$. Interesantes trabajos tanto internacionales como multicéntricos han evaluado sus propias causas de pérdida y posteriormente implementando medidas para mejorar estas cifras ${ }^{7,10,11}$. En su mayoría y en concordancia con nuestra experiencia concluyen que la correcta identificación del médico tratante (pediatra) una vez que el recién nacido es dado de alta, así como entregar una buena educación a los padres, idealmente por escrito, asociado a tener un contacto adecuado y fluido con ellos permite reducir el porcentaje de pérdida. 
A pesar de los esfuerzos y energía puestos para estos fines, sabemos que no es suficiente. Lo ideal sería que ningún niño se perdiera en el seguimiento. En el aproximado $15 \%$ de pérdida a pesar de las medidas implementadas, existen factores que se escapan de nuestro alcance e intervención. Estos factores están relacionados con temas administrativos como la cobertura del seguro catastrófico del paciente en otro centro, lo cual inevitablemente lo hará cambiarse a aquél que se ajuste a su plan. Otro punto de importancia es que al ser Clínica Las Condes un centro de referencia para la atención neonatal avanzada, muchos de los pacientes evaluados y tamizados en UTI neonatal pertenecen originalmente a otros centros y/u otras ciudades y una vez completado su tratamiento son derivados a éstos. De ello se desprende por qué el porcentaje de pérdida de seguimiento en el grupo UTI fue mayor en ambos años estudiados en comparación al grupo sala cuna (42\% v/s $16 \%$ el $2009,27 \%$ v/s $7 \%$ el 2011).

Debemos considerar sin embargo, que al comparar dos cohortes que fueron seguidas en tiempos distintos, nos enfrentamos a la posibilidad de que variables externas a las estudiadas hayan influido positivamente en los resultados, constituyendo un sesgo en el análisis de éstos. Nuestro desafío actual por tanto es investigar cuáles pudiesen ser aquellas variables no consideradas y de esta forma poder estimar su impacto en el seguimiento de los pacientes.

A pesar de todas estas consideraciones una reducción de casi $14 \%$ en la pérdida del seguimiento nos parece una cifra relevante, que se traduce en que aproximadamente de cada 1.000 niños tamizados en el programa sólo se perderán 7 de ellos.

El equipo multidisciplinario compuesto por otorrinolaringólogos, pediatras, neonatólogos al igual que los padres del recién nacido cumplen un rol fundamental en el proceso de evaluación y diagnóstico de estos niños. Sabemos que la realidad nacional no se ajusta a la del centro estudiado, pero teniendo en cuenta que aún no existe tamizaje universal en nuestro país, nos adelantamos a las posibles dificultades que se pueden presentar una vez que estos programas sean implementados. De esta forma hacemos un llamado a que éstos comiencen basados en la experiencia nacional existente y puedan tomar de ella las mejores estrategias con el fin de lograr que todo niño hipoacúsico congénito que nazca en nuestro país tenga las mismas oportunidades diagnósticas y en lo posible terapéuticas.

\section{CONCLUSIONES}

La implementación de medidas prácticas y simples como las que se plantean en este estudio son una forma efectiva para mejorar el seguimiento de los programas de evaluación auditiva neonatal. Sabemos que el diseño y metodología empleados en esta investigación no son los ideales, debido a que se compararon cohortes en tiempos distintos, pero teniendo en cuenta el beneficio otorgado con las estrategias, nos parece éticamente incorrecto que un grupo de recién nacidos no sea sometido a dichas estrategias sólo con el fin de evaluar su impacto.

\section{Agradecimientos}

A Magdalena Castro, Enfermera Epidemióloga de Clínica Las Condes, por su apoyo en el desarrollo metodológico de esta investigación.

\section{BIBLIOGRAFÍA}

1. Yoshinaga-Itano C, Sedey Al, Coulter DK, Mehl Al. Language of early- and later-Identified children with hearing loss. Pediatrics 1998; 102(5): 1161-71.

2. Joint Committe on Infant Hearing. 1994 Position Statement. Disponible en http://www.jcih.org/ JCIH1990.pdf. [Consultado el 8 de agosto de 2012].

3. Joint Committe on Infant Hearing. Year 2000 Position Statement: Principles and Guidelines for Early Hearing Detection and Intervention Programs. Pediatrics 2000; 106 (4): 798-817.

4. Joint Committe on Infant Hearing. Year 2007 Position Statement: Principles and Guidelines for Early Hearing Detection and Intervention Programs. Pediatrics 2007; 120(4): 899-921.

5. Torrente M, Retamal J, Núñez M. Seguimiento audiológico del recién nacido de muy bajo peso. Rev Otorrinolaringol Cir Cabeza Cuello 2007; 67: 115-21. 
6. Nazar G, Goycoolea M, Godoy J, Ried E, Sierra M. Evaluación auditiva neonatal universal: Revisión de 10.000 pacientes estudiados. Rev Otorrinolaringol Cir Cabeza Cuello 2009; 69: 93-102.

7. Russ S, Hanna D, DesGeorges J, Forsman I. Improving Follow-up to Newborn Hearing Screening: A Learning-Collaborative Experience. Pediatrics 2010; 126: S59-S69.

8. Shoup A, Owen K, Jackson G, Laptook A. The parkland memorial hospital experience in ensuring compliance with universal newborn hearing screening follow-up. The Journal of Pediatrics 2005; 146: 66-72.
9. Liu C, Farrell J, MacNeil J, Stone S, Barfield W. Evaluating Loss to Follow-up in Newborn Hearing Screening in Massachusetts. Pediatrics 2008; 121;(2): e335-e343.

10. Shulman S, Besculides M, Saltzman A, Ireys $H$, White K, Forsman I. Evaluation of the Universal Newborn Hearing Screening and Intervention Program. Pediatrics 2010; 126; S19-S27.

11. Deem K, Díaz-Ordaz E, Shiner B. Identifying Quality Improvement Opportunities in a Universal Newborn Hearing Screening Program. Pediatrics 2012; 129: e157-e164. 